\title{
Twist-3 Spin Asymmetries And Parton Correlations In High-energy Processes
}

\author{
Yuji Koike* \\ Department of Physics, Niigata University, Ikarashi 2-no-cho, Nishi-ku, Niigata 950-2181, \\ Japan \\ E-mail: koike@phys.sc.niigata-u.ac.jp
}

In this talk, I present an overview on the study of the twist-3 spin asymmetries based on the collinear factorization. After summarizing the generic feature of the formalism and the twist- 3 distribution and fragmentation functions, I will discuss some of the applications to phenomenological studies on the transverse single-spin asymmetries in the proton-proton collisions at the Relativistic Heavy Ion Collider.

The 26th International Nuclear Physics Conference

11-16 September, 2016

Adelaide, Australia

${ }^{*}$ Speaker. 


\section{Introduction}

Discovery of large transverse single-spin asymmetries (SSA), such as $A_{N}$ in the pion production in proton-proton collision $\left(p^{\uparrow} p \rightarrow \pi X\right)[1,2]$ and hyperon polarization in unpolarized proton proton collision ( $p p \rightarrow \Lambda^{\uparrow} X$ ) [3], triggered lots of theoretical studies on the origin of SSAs. There have been two QCD mechanisms which can account for large SSAs in various processes in $p p$ and $e p$ collisions. One is the "naively $T$-odd" distribution and fragmentation functions in the transverse-momentum-dependent (TMD) factorization [4] which describes SSAs in the small transverse momentum region, $\Lambda_{\mathrm{QCD}} \leq P_{\perp} \ll Q$, where $Q$ is a hard scale in a process and $P_{T}$ is a transverse momentum of the observed hadron. The other is the twist- 3 effect caused by multiparton correlations in the collinear factorization, which describes SSAs at large transverse momentum, $\Lambda_{\mathrm{QCD}} \ll P_{T} \sim Q[5,6]$. It has been also shown that, for Drell-Yan and semi-inclusive deep inealstic scattering (SIDIS), these two mechanisms are equivalent in the intermediate region of the transverse momentum $[7,8,9]$. It is to be noted, however, that the TMD factorization does not hold in general for hadron production in hadron-hadron collisions [10].

These formalisms have been also applied to other spin asymmetries. For example, the double spin asymmetry $A_{L T}$ in the hadron production in the collision between the longitudinally and transversely polarized nucleons, $\vec{p} p^{\uparrow} \rightarrow \pi X$, is also a twist- 3 observable in the collinear factorization.

In this talk, I will discuss recent development in the study of spin observables based on the collinear twist-3 approach. The remainder of this talk is organized as follows: In Sec. 2, I first summarize the fundamental feature of the collinear twist-3 formalism. In Sec. 3, I will discuss some of the recent applications to phenomenology in pp collisions. Sec. IV is a brief summary of this talk.

\section{Twist-3 formalism and multi-parton correlation functions}

\subsection{Three types of twist-3 distribution and fragmentation functions}

We first summarize the distribution and fragmentation functions which are necessary for the calculation of twist- 3 cross sections. The collinear quark distribution functions in the nucleon are defined from the lightcone correlator

$$
\Phi_{i j}(x)=\int_{-\infty}^{\infty} \frac{d \lambda}{2 \pi} \mathrm{e}^{i \lambda x}\left\langle P S\left|\bar{q}_{j}(0)[0 ; \lambda n] q_{i}(\lambda n)\right| P S\right\rangle,
$$

where $q_{i}$ is a quark field with the spinor index $i$, and $|P S\rangle$ is the nucleon state with momentum $P\left(P^{2}=M_{N}^{2}\right)$ and the spin vector $S(P \cdot S=0)$. For $P^{\mu}=(E, \vec{P})$ we define two lightlike vectors $p^{\mu}$ and $n^{\mu}$ as $p^{\mu}=\left(P^{+} / \sqrt{2}\right)(1, \vec{P} /|\vec{P}|)$ and $n^{\mu}=(1,-\vec{P} /|\vec{P}|) /\left(\sqrt{2} P^{+}\right)$with $P^{+}=(E+|\vec{P}|) / \sqrt{2}$, which satisfy $P^{\mu}=p^{\mu}+\left(M_{N}^{2} / 2\right) n^{\mu}$ and $p \cdot n=1$. Decomposition of $\Phi(x)$ defines the quark distributions listed in Table 1 . $[0 ; \lambda n]$ is a gauge link connecting 0 and $\lambda n$. Likewise collinear quark fragmentation functions can be defined from the fragmentation correlator

$$
\Delta_{i j}(z)=\frac{1}{N_{c}} \sum_{X} \int_{-\infty}^{\infty} \frac{d \lambda}{2 \pi} \mathrm{e}^{-i \frac{\lambda}{z}}\left\langle 0\left|[ \pm \infty m ; 0] q_{i}(0)\right| P_{h} S_{h} ; X\right\rangle\left\langle P_{h} S_{h} ; X\left|\bar{q}_{j}(\lambda m)[\lambda m ; \pm \infty m]\right| 0\right\rangle .
$$

The hadron momentum $P_{h}\left(P_{h}^{2}=M_{h}^{2}\right)$ can also be decomposed as $P_{h}^{\mu}=p_{h}^{\mu}+\left(M_{h}^{2} / 2\right) m^{\mu}$ by introducing two lightlike vectors $p_{h}^{\mu}$ and $m^{\mu}$ which are defined similarly to $p^{\mu}$ and $n^{\mu}$. Fragmentation 
functions defined from $\Delta(z)$ are summarized in Table 1. For precise definition of each distribution and fragmentation function, we refer the readers to [11]. We name the twist-3 functions defined from $\Phi(x)$ and $\Delta(z)$ as intrinsic twist-3 distribution/fragmentation functions.

\begin{tabular}{|c|c|c|c|}
\hline $\mathrm{N} \backslash \mathrm{q}$ & Ave. & $S_{\|}$ & $S_{\perp}$ \\
\hline Ave. & $f_{1}(x), e(x)$ & & \\
\hline$S_{\|}$ & & $g_{1}(x)$ & $h_{L}(x)$ \\
\hline$S_{\perp}$ & & $g_{T}(x)$ & $h_{1}(x)$ \\
\hline
\end{tabular}

\begin{tabular}{|c|c|c|c|}
\hline $\mathrm{h} \backslash \mathrm{q}$ & Ave. & $S_{\|}$ & $S_{\perp}$ \\
\hline Ave. & $D(z), E(z), H(z)$ & & \\
\hline$S_{\|}$ & $E_{L}(z)$ & $G_{1}(z)$ & $H_{L}(z)$ \\
\hline$S_{\perp}$ & $D_{T}(z)$ & $G_{T}(z)$ & $H_{1}(z)$ \\
\hline
\end{tabular}

Table 1: Collinear quark distribution functions in the nucleon (left) and quark fragmentation functions for spin-1/2 hadron (right) which are classified by the spin of the hadron and a quark. In the top table $e, h_{L}$ and $g_{T}$ are intrinsic twist- 3 distributions while others are twist- 2 distributions. In the bottom table, $E, H_{L}$ and $G_{T}$ are naively $T$-even intrinsic twist-3 functions, while $H, E_{L}$ and $D_{T}$ are naively $T$-odd intrinsic twist-3 functions. Others are twist-2.

The second type of twist-3 functions are called kinematical ones and are defined from the transverse-momentum-dependent correlators as

$$
\Phi_{\partial, i j}^{\rho}(x)=\int d^{2} k_{T} k_{T}^{\rho} \Phi_{i j}\left(x, k_{T}\right), \quad \Delta_{\partial, i j}^{\rho}(z)=\int d^{2} p_{\perp} p_{\perp}^{\rho} \Delta_{i j}\left(z, p_{\perp}\right),
$$

where $\Phi\left(x, k_{T}\right)$ and $\Delta\left(z, p_{\perp}\right)$ are given as

$$
\begin{aligned}
\Phi_{i j}\left(x, k_{T}\right)= & \int_{-\infty}^{\infty} \frac{d \lambda}{2 \pi} \int \frac{d^{2} z_{T}}{(2 \pi)^{2}} \mathrm{e}^{i x \lambda+i k_{T} \cdot z_{T}}\langle P, S| \bar{q}_{j}(0)[0 ; \infty n]\left[\infty n ; \infty n+z_{T}\right] \\
& \times\left[\infty n+z_{T} ; \lambda n+z_{T}\right] q_{i}\left(\lambda n+z_{T}\right)|P, S\rangle, \\
\Delta_{i j}\left(z, p_{\perp}\right)= & \frac{1}{N_{c}} \sum_{X} \int_{-\infty}^{\infty} \frac{d \lambda}{2 \pi} \int \frac{d^{2} z_{\perp}}{(2 \pi)^{2}} \mathrm{e}^{-i \frac{\lambda}{z}-i p_{\perp} \cdot z_{\perp}}\left\langle 0\left|\left[ \pm \infty m+\infty z_{\perp} ; \pm \infty m\right][ \pm \infty m ; 0] q_{i}(0)\right| P_{h} S_{h} ; X\right\rangle \\
& \times\left\langle P_{h} S_{h} ; X\left|\bar{q}_{j}\left(\lambda m+z_{\perp}\right)\left[\lambda m+z_{\perp} ; \pm \infty m+z_{\perp}\right]\left[ \pm \infty m+z_{\perp} ; \pm \infty m+\infty z_{\perp}\right]\right| 0\right\rangle .
\end{aligned}
$$

Decomposition of $\Phi_{\partial, i j}^{\rho}(x)$ and $\Delta_{\partial, i j}^{\rho}(z)$ defines kinematical twist-3 functions as listed in Table 2.

The third type of the twist-3 functions are called dynamical twist-3 functions and defined from the quark-gluon correlator:

$$
\begin{gathered}
\Phi_{F, i j}^{\rho}\left(x, x^{\prime}\right)=\int_{-\infty}^{\infty} \frac{d \lambda}{2 \pi} \int_{-\infty}^{\infty} \frac{d \mu}{2 \pi} \mathrm{e}^{i x^{\prime} \lambda+i\left(x-x^{\prime}\right) \mu}\left\langle P, S\left|\bar{q}_{j}(0)[0 ; \mu n] \operatorname{ign}_{\eta} F^{\eta \rho}(\mu n)[\mu n ; \lambda n] q_{i}(\lambda n)\right| P, S\right\rangle, \\
\Delta_{F, i j}^{\rho}\left(z, z^{\prime}\right)=\frac{1}{N_{c}} \sum_{X} \int_{-\infty}^{\infty} \frac{d \lambda}{2 \pi} \int_{-\infty}^{\infty} \frac{d \mu}{2 \pi} \mathrm{e}^{i \frac{\lambda}{z^{+}}+i\left(\frac{1}{z}-\frac{1}{z}\right) \mu}\langle 0|[ \pm \infty m ; \mu m] \operatorname{igm}_{\eta} F^{\eta \rho}(\mu m) \\
\times[\mu m ; \lambda m] q_{i}(\lambda m)\left|P_{h} S_{h} ; X\right\rangle\left\langle P_{h} S_{h} ; X\left|\bar{q}_{j}(0)[0 ; \pm \infty m]\right| 0\right\rangle .
\end{gathered}
$$

Decomposition of $\Phi_{F, i j}^{\rho}\left(x, x^{\prime}\right)$ and $\Delta_{F, i j}^{\rho}\left(z, z^{\prime}\right)$ defines the dynamical twist-3 functions as listed in Table 3. The dynamical twist-3 distribution functions are real, and have definite symmetry under $x \leftrightarrow x^{\prime}: H_{F U}$ and $F_{F T}$ are symmetric, while $H_{F L}$ and $G_{F T}$ are anti-symmetric. The dynamical twist3 fragmentation functions are complex functions: The real part is naively $T$-even, while imaginary part is naively $T$-odd. Neither real nor imaginary parts have definite symmetry properties. The support of dynamical twist-3 functions is $|x|<1,\left|x^{\prime}\right|<1,\left|x^{\prime}-x\right|<1$ for distribution functions and $0<z<1, z<z^{\prime}<\infty$ for the fragmentation functions. 


\begin{tabular}{|c|c|c|c|}
\hline $\mathrm{N} \backslash \mathrm{q}$ & Ave. & $S_{\|}$ & $S_{\perp}$ \\
\hline Ave. & & & $h_{1}^{\perp(1)}(x)$ \\
\hline$S_{\|}$ & & & $h_{1 L}^{\perp(1)}(x)$ \\
\hline$S_{\perp}$ & $f_{1 T}^{\perp(1)}(x)$ & $g_{1 T}^{(1)}(x)$ & \\
\hline
\end{tabular}

\begin{tabular}{|c|c|c|c|}
\hline $\mathrm{h} \backslash \mathrm{q}$ & Ave. & $S_{\|}$ & $S_{\perp}$ \\
\hline Ave. & & & $H_{1}^{\perp(1)}(z)$ \\
\hline$S_{\|}$ & & & $H_{1 L}^{\perp(1)}(z)$ \\
\hline$S_{\perp}$ & $D_{1 T}^{\perp(1)}(z)$ & $G_{1 T}^{(1)}(z)$ & \\
\hline
\end{tabular}

Table 2: Kinematical twist-3 distribution functions (left) and fragmentation functions (right) classified by the spin of the hadron and a quark.

\begin{tabular}{|c|c|}
\hline Ave. & $H_{F U}\left(x_{1}, x_{2}\right)$ \\
\hline$S_{\|}$ & $H_{F L}\left(x_{1}, x_{2}\right)$ \\
\hline$S_{\perp}$ & $F_{F T}\left(x_{1}, x_{2}\right), G_{F T}\left(x_{1}, x_{2}\right)$ \\
\hline
\end{tabular}

\begin{tabular}{|c|c|}
\hline Ave. & $\widehat{H}_{F U}\left(z_{1}, z_{2}\right)$ \\
\hline$S_{\|}$ & $\widehat{H}_{F L}\left(z_{1}, z_{2}\right)$ \\
\hline$S_{\perp}$ & $\widehat{D}_{F T}\left(z_{1}, z_{2}\right), \widehat{G}_{F T}\left(z_{1}, z_{2}\right)$ \\
\hline
\end{tabular}

Table 3: Dynamical twist-3 distribution functions (left) and fragmentation functions (right) classified by the hadron spin configuration.

\subsection{Structure of the twist-3 cross section}

\subsubsection{Naively $T$-odd observables}

For naively $T$-odd observables like SSAs, the cross section occurs from an interference between amplitudes which have different complex phases. This leads to the following features in the cross sections.

(i) For the contribution from the twist- 3 distribution, the cross section can be written solely by using the dynamical twist- 3 distribution functions and the corresponding partonic cross section is caused from the imaginary part ( $\delta$-function piece) of an internal propagator in the hard part $[5,6]$. This pole contribution fixes the momentum fraction in the dynamical distribution $F\left(x_{1}, x_{2}\right)$ as either $x_{1}=x_{2}, x_{i}=0(i=1$ or 2$)$ or $x_{i} \neq 0$ ( $i=1$ or 2$)$, which are often called soft-gluon-pole (SGP), soft-fermion-pole (SFP) or hard-pole (HP), respectively. In the LO spin-dependent cross sections for $p^{\uparrow} p \rightarrow h X(h=\pi, \gamma)[12,13,14]$ and $p p \rightarrow \Lambda^{\uparrow} X$ [15], only massless particles (quark, gluon and photon) participate in the hard scattering, and thus only SGP and SFP appear. For Drell-Yan $\left(p^{\uparrow} p \rightarrow \gamma^{*} X\right)$ and SIDIS ( $\left.e p^{\uparrow} \rightarrow e h X\right)$, HP as well as SGP and SFP contribute [6, 7].

(ii) For the twist-3 fragmentation contribution, naively $T$-odd intrinsic and kinematical functions and the imaginary part of the dynamical functions are convoluted with the nonpole piece of the partonic hard scattering part to give rise to the cross section $[9,16,17]$.

\subsubsection{Naively $T$-even observables}

For such observables like $A_{L T}$ in hadron production, $\vec{\ell} p^{\uparrow} \rightarrow \pi X$ [18], $\vec{p} p^{\uparrow} \rightarrow \pi X[19,20]$, both twist- 3 distribution and fragmentation functions appear in the cross section as nonpole contributions. In particular, the real part of the dynamical twist-3 fragmentation functions contribute. Therefore $T$-even and $T$-odd observables probe different aspects of the collinear twist- 3 formalism and the hadron structure.

\subsection{Operator constraints for the twist-3 functions}

The twist-3 functions given in Tables 1-3 are not independent but obey constraint relations which follow from the QCD equation of motion (EOM) and the Lorentz invariance property of 
the correlator for each distribution and fragmentation function. The latter is a nonlocal version of the operator product expansion (OPE). In [11], we derived the complete set of those relations, in particular, those for the twist-3 fragmentation functions are new. Here we list the results.

\subsubsection{EOM relations for twist-3 fragmentation functions}

(i) Unpolarized hadron:

$$
\begin{aligned}
& H_{1}^{\perp(1)}(z)=-\frac{H(z)}{2 z}+\int_{z}^{\infty} \frac{d z^{\prime}}{z^{\prime 2}} \frac{\mathfrak{I}\left[\hat{H}_{F U}\left(z, z^{\prime}\right)\right]}{\frac{1}{z}-\frac{1}{z^{\prime}}}, \\
& -\frac{E(z)}{2 z}=\int_{z}^{\infty} \frac{d z^{\prime}}{z^{\prime 2}} \frac{\Re\left[\hat{H}_{F U}\left(z, z^{\prime}\right)\right]}{\frac{1}{z}-\frac{1}{z^{\prime}}}-\frac{m_{q}}{2 M_{h}} D_{1}(z),
\end{aligned}
$$

where $\mathfrak{I}$ and $\mathfrak{R}$ represent imaginary and real parts, respectively.

(ii) Transversely polarized spin- $1 / 2$ hadron:

$$
\begin{aligned}
& D_{1 T}^{\perp(1)}(z)=-\frac{D_{T}(z)}{z}+\int_{z}^{\infty} \frac{d z^{\prime}}{z^{\prime 2}} \frac{\mathfrak{I}\left[\hat{D}_{F T}\left(z, z^{\prime}\right)\right]-\mathfrak{I}\left[\hat{G}_{F T}\left(z, z^{\prime}\right)\right]}{\frac{1}{z}-\frac{1}{z^{\prime}}} \\
& G_{1 T}^{(1)}(z)=\frac{G_{T}(z)}{z}-\frac{m_{q}}{M_{h}} H_{1}(z)+\int_{z}^{\infty} \frac{d z^{\prime}}{z^{\prime 2}} \frac{\mathfrak{R}\left[\hat{D}_{F T}\left(z, z^{\prime}\right)\right]-\mathfrak{R}\left[\hat{G}_{F T}\left(z, z^{\prime}\right)\right]}{\frac{1}{z}-\frac{1}{z^{\prime}}} .
\end{aligned}
$$

(iii) Longitudinally polarized spin-1/2 hadron:

$$
\begin{aligned}
& \frac{E_{L}(z)}{2 z}=-\int_{z}^{\infty} \frac{d z^{\prime}}{z^{\prime 2}} \frac{\mathfrak{J}\left[\hat{H}_{F L}\left(z, z^{\prime}\right)\right]}{\frac{1}{z}-\frac{1}{z^{\prime}}}, \\
& H_{1 L}^{\perp(1)}(z)=-\frac{H_{L}(z)}{2 z}+\frac{m_{q}}{2 M_{h}} G_{1}(z)-\int_{z}^{\infty} \frac{d z^{\prime}}{z^{\prime 2}} \frac{\Re\left[\hat{H}_{F L}\left(z, z^{\prime}\right)\right]}{\frac{1}{z}-\frac{1}{z^{\prime}}} .
\end{aligned}
$$

\subsubsection{Lorentz invariance relations for twist-3 fragmentation functions}

In this subsection, we list the relations which follows from the Lorentz invariance of the correlators. We present the relations in the form of the so-called Lorentz invariance relations (LIR) which are obtained by combining the OPE relations with the EOM relations.

(i) Unpolarized hadron:

$$
\frac{H(z)}{z}=-\left(1-z \frac{d}{d z}\right) H_{1}^{\perp(1)}(z)-\frac{2}{z} \int_{z}^{\infty} \frac{d z^{\prime}}{z^{\prime 2}} \frac{\mathfrak{I}\left[\hat{H}_{F U}\left(z, z^{\prime}\right)\right]}{\left(1 / z-1 / z^{\prime}\right)^{2}} .
$$

(ii) Transversely polarized spin-1/2 hadron:

$$
\begin{aligned}
& \frac{G_{T}(z)}{z}=\frac{G_{1}(z)}{z}+\left(1-z \frac{d}{d z}\right) G_{1 T}^{(1)}(z)-\frac{2}{z} \int_{z}^{\infty} \frac{d z^{\prime}}{z^{\prime 2}} \frac{\mathfrak{R}\left[\hat{G}_{F T}\left(z, z^{\prime}\right)\right]}{\left(1 / z-1 / z^{\prime}\right)^{2}}, \\
& \frac{D_{T}(z)}{z}=-\left(1-z \frac{d}{d z}\right) D_{1 T}^{\perp(1)}(z)-\frac{2}{z} \int_{z}^{\infty} \frac{d z^{\prime}}{z^{\prime 2}} \frac{\mathfrak{I}\left[\hat{D}_{F T}\left(z, z^{\prime}\right)\right]}{\left(1 / z-1 / z^{\prime}\right)^{2}} .
\end{aligned}
$$

(iii) Longitudinally polarized spin-1/2 hadron:

$$
\frac{H_{L}(z)}{z}=\frac{H_{1}(z)}{z}-\left(1-z \frac{d}{d z}\right) H_{1 L}^{\perp(1)}(z)+\frac{2}{z} \int_{z}^{\infty} \frac{d z^{\prime}}{z^{\prime 2}} \frac{\Re\left[\hat{H}_{F L}\left(z, z^{\prime}\right)\right]}{\left(1 / z-1 / z^{\prime}\right)^{2}},
$$


Using the EOM relations and LIRs, one can eliminate the intrinsic and kinematical twist-3 functions in favor of the dynamical twist- 3 functions and the twist- 2 functions. In this sense the intrinsic and kinematical twist-3 functions may be regarded auxiliary functions. On the other hand, in the leading-order calculation, simple structure of partonic hard cross sections sometimes leads to a compact formula for the twist- 3 cross sections in terms of the intrinsic and kinematical twist- 3 functions [20].

\subsubsection{Lorentz invariance of the twist-3 cross sections}

Twist-3 cross sections can be obtained as a convolution of the hadronic correlators introduced in Sec. 2.1 and the corresponding hard part. The resulting twist-3 cross sections involve intrinsic, kinematical and dynamical twist-3 distribution and fragmentation functions together with twist-2 functions. In the decomposition of the hadronic correlators, the coefficients of the twist- 3 functions contain lightlike vectors $n$ and $m$, and thus the expression for the cross sections also contain these vectors. On the other hand, the cross section should have a Lorentz invariant expression in terms of only the physical vectors such as momenta of the particles participating in the process and the spin vectors. Though $n$ and $m$ are uniquely determined from $P$ and $P_{h}$, their expression in terms of the physical vectors depends on a frame we choose, which makes the frame-independence of the cross section unclear.

In [11] (see also [18]), we investigated this point, using, as an example, the inclusive hadron production in the lepton-nucleon collision, $e(l)+P(P) \rightarrow h\left(P_{h}\right)+X$. We define Mandelstam variables for this process as $S=(P+l)^{2}, T=\left(P-P_{h}\right)^{2}$ and $U=\left(l-P_{h}\right)^{2}$. In the cross section, $l, P$ and $P_{h}$ can be regarded as lightlike in the twist-3 accuracy, and therefore $P$ and $P_{h}$ can be identified as $p$ and $p_{h}$, respectively. In the $e N$ center-of-mass frame, $n^{\mu}=2 l^{\mu} / S$. In the $h N$ center-of-mass frame, $n^{\mu}=-2 P_{h}^{\mu} / T$ and $m^{\mu}=-2 P^{\mu} / T$. After obtaining the twist- 3 cross sections in terms of $n$ and $m$, one can eliminate them in terms of the physical momenta. For $e N^{\uparrow} \rightarrow h X, \vec{e} N^{\uparrow} \rightarrow h X$, $e N \rightarrow \Lambda^{\uparrow} X$ and $\vec{e} N \rightarrow \Lambda^{\uparrow} X$, we have shown that the EOM relations and LIRs, and

$$
\pi F_{F T}(x, x)=f_{1 T}^{\perp(1)}(x), \quad \pi H_{F U}(x, x)=h_{1}^{\perp(1)}(x),
$$

lead to frame-independent expression for the twist- 3 cross sections in terms of the physical momenta.

\section{Twist-3 phenomenology for SSA at RHIC}

In this section, as an application of the twist-3 analysis to phenomenology, I will discuss $A_{N}$ in inclusive pion production and direct photon production in $p p$ collisions at Relativistic Heavy Ion Collider (RHIC) of Brookhaven National Laboratory. For $p p \rightarrow \pi X$, the next-to-leading order (NLO) perturbative QCD can describe the unpolarized cross section very well [21]. Therefore, as a first step, it makes sense to apply the fixed-order perturbative QCD in the collinear factorization formalism to the polarized cross section. We also mention it's been known that the TMD factorization is not valid for inclusive hadron production in pp collisions. 


\section{1 $A_{N}$ for $p^{\uparrow} p \rightarrow \pi X$ with twist-3 fragmentation}

Three kinds of twist- 3 cross sectoins contribute to the single-spin-dependent cross section for $p^{\uparrow} p \rightarrow \pi X:$

$$
\begin{aligned}
& \Delta \sigma \sim\left\{F_{F T}, G_{F T}\right\}\left(x_{1}, x_{2}\right) \otimes f\left(x^{\prime}\right) \otimes D(z) \otimes \hat{\sigma}_{A}+G_{F}^{3 g}\left(x_{1}, x_{2}\right) \otimes f\left(x^{\prime}\right) \otimes D(z) \otimes \hat{\sigma}_{B} \\
& +h_{1}(x) \otimes f\left(x^{\prime}\right) \otimes\left\{H(z), H_{1}^{\perp(1)}(z), \widehat{H}_{F U}\left(z_{1}, z_{2}\right)\right\} \otimes \hat{\sigma}_{C}+h_{1}(x) \otimes H_{F U}\left(x_{1}^{\prime}, x_{2}^{\prime}\right) \otimes D(z) \otimes \hat{\sigma}_{D},
\end{aligned}
$$

where the first, second and third nonperturbative function in each term represents the distribution function in the polarized nucleon, the distribution function in the unpolarized nucleon, and the fragmentation function for the final pion, and $\sigma_{A, B, C, D}$ stand for the corresponding partonic hard factor. $G_{F}^{3 g}$ is the three-gluon correlation function in the polarized nucleon. There had been several analyses by assuming the 1 st term is the main origin of the observed asymmetry $[12,13,22]$. However, it's been pointed out that the resulting SGP function $F_{F T}(x, x)$ (Note $G_{F T}(x, x) \equiv 0$ by symmetry) has a sign opposite to what is expected from the analysis of SSAs observed in SIDIS [23], which is known as the "sign mismatch" problem. The 4th term was studied in [24] and it was shown that the partonic hard cross section $\sigma_{D}$ is negligible $\left(\sigma_{D} \ll \sigma_{A}\right)$. The 2 nd term was analyzed in [25], and it was shown that it can't be a main origin of the asymmetry since the pattern of $A_{N}$ for $\pi^{ \pm, 0}$ caused by $G_{F}^{3 g}$ is not consistent with the observed one. Under this circumstance, the remaining sources could be the 1st and 3rd terms in (3.1). The LO cross section for the 3rd term was derived in [26], and a numerical analysis of the RHIC $A_{N}$ data for the pion production was performed in [27] by including the 1 st and 3 rd terms. In this analysis, the SGP function $F_{F T}(x, x)$ was fixed by the Sivers function obtained from the analysis of the SSA in SIDIS through the relation (2.18). The cross section for the 3rd term reads [26]

$$
\begin{aligned}
& E_{h} \frac{d \sigma\left(\vec{S}_{\perp}\right)}{d^{3} \vec{P}_{h}}=- \frac{2 \alpha_{s}^{2} M_{h}}{S} \varepsilon_{\perp, \alpha \beta} S_{\perp}^{\alpha} P_{h \perp}^{\beta} \sum_{i} \sum_{a, b, c} \int_{z_{\min }}^{1} \frac{d z}{z^{3}} \int_{x_{\min }^{\prime}}^{1} \frac{d x^{\prime}}{x^{\prime}} \frac{1}{x} \frac{1}{x^{\prime} S+T / z} \frac{1}{-x^{\prime} \hat{t}-x \hat{u}} \\
& \times h_{1}^{a}(x) f_{1}^{b}\left(x^{\prime}\right)\left[\left(\hat{H}^{\pi / c}(z)-z \frac{d \hat{H}^{\pi / c}(z)}{d z}\right) S_{\hat{H}^{i}}^{i}+\frac{1}{z} H^{\pi / c}(z) S_{H}^{i}\right. \\
&\left.+2 z^{2} \int_{z}^{\infty} \frac{d z_{1}}{z_{1}^{2}} \frac{1}{1 / z-1 / z_{1}} \operatorname{Im} \hat{H}_{F U}^{\pi / c}\left(z, z_{1}\right) \frac{1}{1-z / z_{1}} S_{\hat{H}_{F U}}^{i}\right]
\end{aligned}
$$

where $\hat{H}(z)\left(\sim H_{1}^{\perp(1)}(z)\right)$ is given in terms of the Collins function $H_{1}^{\perp}$ as

$$
\hat{H}^{\pi / c}(z)=z^{2} \int d^{2} \vec{k}_{\perp} \frac{\vec{k}_{\perp}^{2}}{2 M_{h}^{2}} H_{1}^{\perp \pi / c}\left(z, z^{2} \vec{k}_{\perp}^{2}\right) .
$$

The intrinsic twist-3 fragmentation function $H(z)$ is determined from $H_{1}^{\perp(1)}(z)$ and the dynamical twist-3 fragmentation function $\hat{H}_{F U}\left(z, z_{1}\right)$ by the EOM relation (2.8). In [27], $H_{1}^{\perp(1)}(z)$ and the transversity distribution $h_{1}(x)$ in (3.2) were fixed by the hadron production in $e^{+} e^{-}$-annihilation and SIDIS, and $\hat{H}_{F U}\left(z, z_{1}\right)$ was determined by fitting to the RHIC $A_{N}$ data. We recall that with the SGP function determined by (2.18) the first term in (3.1) gives small $A_{N}$ with opposite sign 
("sign mismatch"). The result shows that $\hat{H}_{F U}\left(z, z_{1}\right)$ plays a crucial role to reproduce the data: If one sets $\hat{H}_{F U}\left(z, z_{1}\right) \equiv 0$ in (3.2) and the EOM relation (2.8), one can not obtain an appropriate magnitude for $A_{N}$ although the remaining contribution in (3.2) gives the correct sign for $A_{N}$. It was found that nonzero $\hat{H}_{F U}\left(z, z_{1}\right)$ can reproduce experimental $A_{N}$, which shows that the fragmentation contribution may be a main cause of the observed $A_{N}$ at RHIC. In this analysis, however, the LIR (2.14) was not taken into account. Accordingly, to draw a definite conclusion on the role of the fragmetation contribution, one needs to reanalyze the data, taking this relation into account.

\section{$3.2 A_{N}$ for $p^{\uparrow} p \rightarrow \gamma X$}

The direct photon production is free from fragmentation ambiguity, and thus it is an ideal process to investigate the nucleon structure. The twist- 3 spin-dependent cross section for $p^{\uparrow} p \rightarrow \gamma X$ consists of three parts:

$$
\Delta \sigma \sim\left\{F_{F T}, G_{F T}\right\}\left(x_{1}, x_{2}\right) \otimes f\left(x^{\prime}\right) \otimes \hat{\sigma}_{A}+G_{F}^{3 g}\left(x_{1}, x_{2}\right) \otimes f\left(x^{\prime}\right) \otimes \hat{\sigma}_{B}+h_{1}(x) \otimes H_{F U}\left(x_{1}^{\prime}, x_{2}^{\prime}\right) \otimes \hat{\sigma}_{C}
$$

The LO SGP and SFP cross sections for the 1st term were derived in [12,14] and [28], respectively, and their effect on $A_{N}$ at RHIC energy was studied in [29]. It shows that the SFP contribution is negligible compared to that from SGP, and the latter can cause nonzero $A_{N}$ only at $x_{F}>0$, i.e., direct photon production in the forward hemisphere with respect to the polarized proton. The cross section for the 2nd term was derived in [30], which shows that the 2nd term can cause nonzero asymmetry only in the backward direction $\left(x_{F}<0\right)$ and its magnitude is very sensitive to the small$x$ behavior of the three-gluon correlation function. The cross section for the 3rd term was derived in [31], which shows that the SFP cross section is identically zero and the SGP contribution is also negligible compared with the 1st line. From these studies, one can conclude that the SGP component of the 1 st line is the only source for $A_{N}^{\gamma}$ at $x_{F}>0$, while the three-gluon correlation (2nd term) is the only one for $A_{N}^{\gamma}$ at $x_{F}<0$. Therefore, $A_{N}^{\gamma}$ plays an important role to determine these correlation functions.

\section{Summary}

In this talk, I have summarized some of the recent develoment in the study of spin observables based on the collinear twist-3 approach. The LO cross section formula have been derived for most of the relevant processes in ep and pp collisions. The next step will be an inclusion of NLO corrections, which enables more realistic study on the RHIC data as well as future Electoron Ion Collider experiment.

\section{Acknowledgments}

This work has been supported by the Grants-in-Aid for Scientific Research from the Japan Society of Promotion of Science under Contract No. 26287040. 


\section{References}

[1] D. L. Adams et al. [E581 and E704 Collaborations], Phys. Lett. B 261, 201 (1991); D. L. Adams et al. [E704 Collaboration], Phys. Lett. B 264, 462 (1991).

[2] J. Adams et al. [STAR Collaboration], Phys. Rev. Lett. 92, 171801 (2004); B. I. Abelev et al. [STAR Collaboration], Phys. Rev. Lett. 101, 222001 (2008).

[3] G. Bunce et al., Phys. Rev. Lett. 36, 1113 (1976).

[4] J. Collins, "Foundations of perturbative QCD," (Cambridge monographs on particle physics, nuclear physics and cosmology. 32)

[5] J. w. Qiu and G. F. Sterman, Phys. Rev. Lett. 67, 2264 (1991); Nucl. Phys. B 378, 52 (1992).

[6] H. Eguchi, Y. Koike and K. Tanaka, Nucl. Phys. B 763, 198 (2007).

[7] X. Ji, J. W. Qiu, W. Vogelsang and F. Yuan, Phys. Rev. Lett. 97, 082002 (2006); Phys. Rev. D 73, 094017 (2006); Phys. Lett. B 638, 178 (2006).

[8] Y. Koike, W. Vogelsang and F. Yuan, Phys. Lett. B 659, 878 (2008).

[9] F. Yuan and J. Zhou, Phys. Rev. Lett. 103, 052001 (2009).

[10] T. C. Rogers and P. J. Mulders, Phys. Rev. D 81, 094006 (2010).

[11] K. Kanazawa, Y. Koike, A. Metz, D. Pitonyak and M. Schlegel, Phys. Rev. D 93, 054024 (2016).

[12] C. Kouvaris, J. W. Qiu, W. Vogelsang and F. Yuan, Phys. Rev. D 74, 114013 (2006).

[13] Y. Koike and T. Tomita, Phys. Lett. B 675, 181 (2009).

[14] Y. Koike and K. Tanaka, Phys. Rev. D 76, 011502 (2007).

[15] Y. Kanazawa and Y. Koike, Phys. Rev. D 64, 034019 (2001); J. Zhou, F. Yuan and Z.-T. Liang, Phys. Rev. D 78, 114008 (2008); Y. Koike, K. Yabe and S. Yoshida, Phys. Rev. D 92, 094011 (2015).

[16] Z. B. Kang, F. Yuan and J. Zhou, Phys. Lett. B 691, 243 (2010).

[17] K. Kanazawa and Y. Koike, Phys. Rev. D 88, 074022 (2013).

[18] K. Kanazawa, A. Metz, D. Pitonyak and M. Schlegel, Phys. Lett. B 742, 340 (2015).

[19] A. Metz, D. Pitonyak, A. Schäfer and J. Zhou, Phys. Rev. D 86, 114020 (2012).

[20] Y. Koike, D. Pitonyak, Y. Takagi and S. Yoshida, Phys. Lett. B 752, 95 (2016); Y. Koike, D. Pitonyak and S. Yoshida, Phys. Lett. B 759, 75 (2016).

[21] B. Jager, A. Schafer, M. Stratmann and W. Vogelsang, Phys. Rev. D 67, 054005 (2003).

[22] K. Kanazawa and Y. Koike, Phys. Rev. D 82, 034009 (2010). Phys. Rev. D 83, 114024 (2011).

[23] Z. B. Kang, J. W. Qiu, W. Vogelsang and F. Yuan, Phys. Rev. D 83, 094001 (2011).

[24] Y. Kanazawa and Y. Koike, Phys. Lett. B 478, 121 (2000); Phys. Lett. B 490, 99 (2000).

[25] H. Beppu, K. Kanazawa, Y. Koike and S. Yoshida, Phys. Rev. D 89, 034029 (2014).

[26] A. Metz and D. Pitonyak, Phys. Lett. B 723, 365 (2013).

[27] K. Kanazawa, Y. Koike, A. Metz and D. Pitonyak, Phys. Rev. D 89, no. 11, 111501 (2014).

[28] K. Kanazawa and Y. Koike, Phys. Lett. B 701, 576 (2011).

[29] K. Kanazawa and Y. Koike, Phys. Lett. B 720, 161 (2013).

[30] Y. Koike and S. Yoshida, Phys. Rev. D 85, 034030 (2012).

[31] K. Kanazawa, Y. Koike, A. Metz and D. Pitonyak, Phys. Rev. D 91, no. 1, 014013 (2015). 